Revue d'histoire de l'enfance « irrégulière »

Le Temps de l'histoire

$3 \mid 2000$

L'enfant de justice pendant la guerre et l'immédiat après-guerre

\title{
René Biard, Claude Charmes, deux « enfants de justice » pendant la guerre
}

Jacques Bourquin

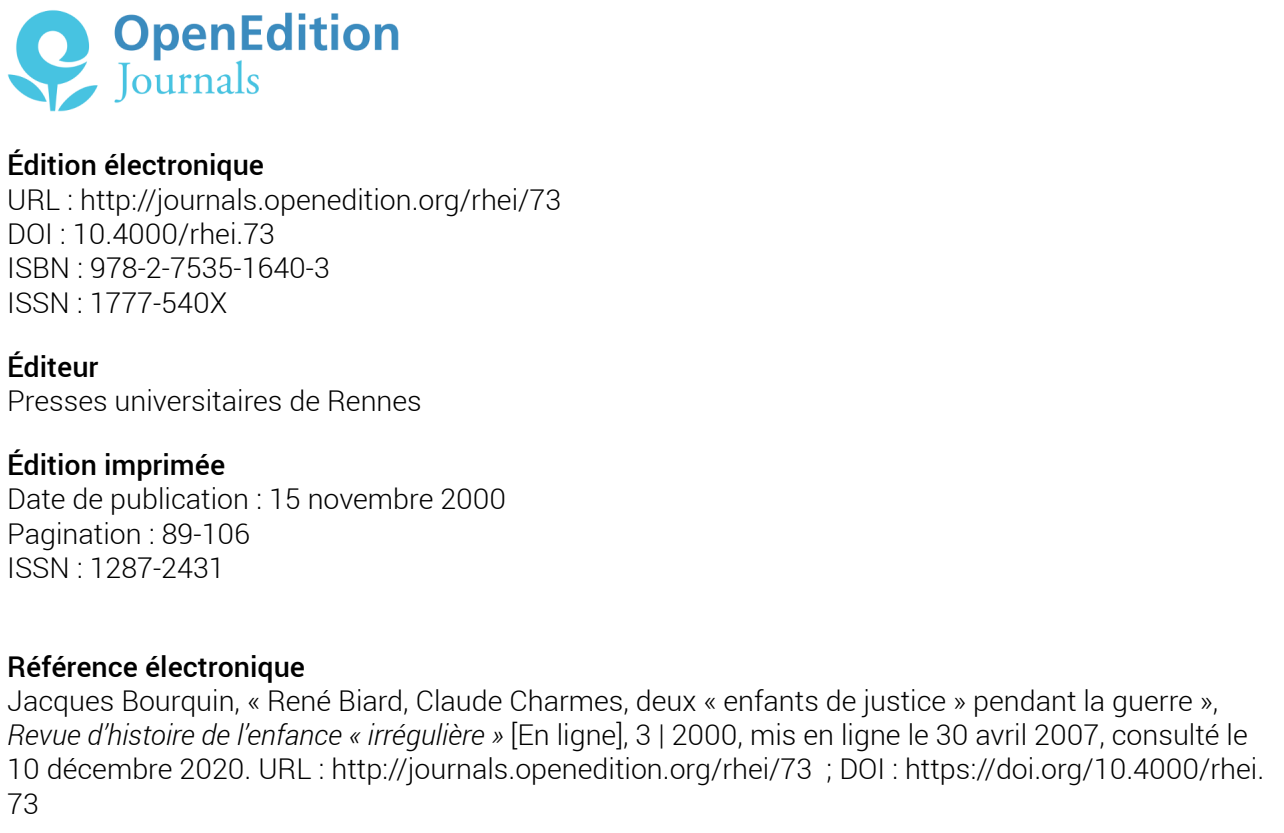

(c) PUR 


\section{René Biard, Claude Charmes, deux "enfants de justice" pendant la guerre}

\section{I - Introduction}

L'enfant de justice pendant la guerre, il faudrait le découvrir à partir d'une analyse de ces très nombreux dossiers de mineurs qui ont été constitués dans les centres d'accueil et les centres d'observation et de triage apparus dans l'application de la loi du 27 juillet 1942 sur l'enfance délinquante. Il y a là une sorte de regard kaléidoscopique sur ce mineur, celui du policier, du juge, du médecin, de l'assistante sociale et de ce nouveau venu qu'est l'éducateur. Tous ces regards s'additionnent dans une optique de classification, de triage, mot clé de l'époque, où il s'agit de distinguer, parmi ces mineurs, ceux qui sont rééducables de ceux qui ne le sont pas. Il y a là un travail qui devrait intéresser les historiens; les archives sont nombreuses et relativement accessibles.

Notre projet, bien moins ambitieux, est tout autre. Nous avons recherché des récits autobiographiques de ces mineurs, qui aient fait l'objet de publication, des témoignages dans leur subjectivité, leurs zones d'ombre, leurs enluminures, leurs oublis volontaires ou non, tout en sachant que ces récits avaient été le plus souvent écrits et publiés plus de vingt ans après les faits.

Les années 1970-1980 nous paraissaient avoir été relativement prolixes en ouvrages autobiographiques sur la prison, sur les maisons de correction. Dans la foulée des travaux de Michel Foucault sur la peine, sur l'enfermement, dans la militance qui s'en suivit avec la création du groupe d'information sur les prisons, ces témoignages étaient recherchés et souvent préfacés par des intellectuels. Michel Foucault avait préfacé, en 1973, De la prison à la révolte de Serge Livrozet ; Claude Mauriac avait fait de même, en 1980, avec L'acharnement de Roger Knobelspiess.

Jacques Bourquin / pp. 89 à 106 
(1) René BIARD,

Bagnards en culottes courtes, Paris, La Table ronde, 1968, p. 9.

(2) Philippe LEJEUNE, Le pacte autobiographique, Paris, Le Seuil, 1975, p. 264.
Le problème des maisons de correction, dont Michel Foucault avait soulevé le voile avec Surveiller et Punir, intéressait aussi les éditeurs et de nombreux anciens colons publièrent leurs souvenirs : Jean-Guy Le Dano, René Biard, Claude Charmes, Alain Kerdavid, Henri le Lyonnais... Ils avaient eu quelques devanciers célèbres, entrés dans le patrimoine littéraire : Jean Genet, qui évoquait en 1944, dans le magnifique Miracle de la Rose, son séjour à la Colonie de Mettray à la fin des années 1920, et Albertine Sarrasin, qui construisait une œuvre à partir de son passage à Fresnes chez les mineures au cours des années 1950. Seuls René Biard, auteur de Bagnards en culottes courtes, publié à la Table ronde en 1968, et Claude Charmes avec Le maximum, publié chez Stock en 1974, évoquaient en tant que mineurs la période de la guerre. Ils sont nos deux témoins privilégiés.

Mais quel statut devons-nous donner à leurs écrits ? Ni l'un, ni l'autre ne sont des professionnels de l'écriture.

René Biard, dont c'est le premier ouvrage, nous laisse entendre qu'il prévoit d'autres publications ; nous n'en avons pas retrouvé la trace. Il apparaît toutefois comme le seul auteur de son autobiographie. Son préfacier, A. Legouy, aumônier des prisons, nous confirme dans cette idée lorsqu'il écrit : " R. Biard écrit comme il parle, il parle comme il a appris à parler dans le monde où il a vécu... c'est une langue vivante ». (1) On sent, à la lecture de l'ouvrage, combien l'auteur est influencé par la langue des truands, celle d'Albert Simonin, célèbre auteur de romans policiers de l'époque.

Le cas de Claude Charmes est différent. Si le nom d'aucun coauteur ne figure sur la couverture, la page de titre à l'intérieur du livre mentionne "souvenirs recueillis par Jacques Perrier ". C'est ce que Philippe Lejeune appelle " une autobiographie parlée ", " une parole avant l'écriture ». ${ }^{(2)}$ Le récit de vie a une origine orale ; c'est un moyen de faire l'autobiographie de ceux qui n'écrivent pas, technique ethnographique largement utilisée par la collection "Terres humaines", chez Plon, depuis une trentaine d'années. On se souvient aussi de la parution au Seuil, en 1996, de Grenadou paysan français, fruit d'une collaboration entre l'autobiographe Emphraïm Grenadou et un journaliste, Alain Prévost. Les 
exemples se multiplient pendant les années 1970 ; les éditeurs s'empressent d'exploiter cette nouvelle demande du public, friand de témoignages, de récits de vie.

Qu'en est-il pour Claude Charmes ? Le synopsis de son livre nous apprend que, lors de sa libération en juillet 1974, il est passé dans une émission de télévision, alors qu'on est en pleine période de révoltes des prisons. L'émission a un fort impact. Claude Charmes est sollicité pour des interviews à Hambourg, à Londres, à New York. Il entre dans l'actualité internationale. L'idée d'un livre est lancée. Il faut faire vite. D'où vraisemblablement le recours à Jacques Perrier. Le maximum sort au dernier trimestre 1974. Le livre a du succès, car il est réédité l’année suivante au "Club pour vous", chez Hachette, dont la diffusion est très large. On peut supposer que l'auteur, en raison de son niveau d'études - à sa sortie de prison, il prépare un doctorat en droit - aurait pu se passer de l'aide de J. Perrier, mais n'était-il pas plus simple, sur le plan psychologique, de raconter à un autre une histoire douloureuse plutôt que de l'écrire soi-même.

Deux auteurs, deux statuts d'écriture différents.

\section{II - Un contexte familial difficile}

René Biard a 15 ans en 1940, Claude Charmes en a 8 : un adolescent, un enfant. Tous deux ont fait, dès leur prime enfance, l'objet de multiples placements chez des nourrices, dans des institutions. On est avec eux dans l'univers des familles dissociées, parents divorcés ou séparés ; l'un comme l'autre apparaissent comme une parfaite illustration des thèses de l'époque dans le secteur de l'enfance irrégulière ou déficiente : le lien entre la délinquance juvénile, l'inadaptation sociale et la dissociation familiale, la guerre intervenant comme une cause supplémentaire et non négligeable.

Le Dr Georges Heuyer, un des pionniers de la neuropsychiatrie infantile qui aura un rôle important dans la constitution du secteur de l'enfance inadaptée pendant la guerre, écrit dans un rapport qu'il rédigea en 1947 sur les enfants victimes de la guerre : "La dissociation familiale est le facteur le plus important des troubles psychiques de l'enfance et de la jeunesse, en 
(3) Georges HEUYER, "Psychopathologie de l'enfant victime de la guerre", Revue de la sauvegarde, janvier 1948, p. 5 .

(4) Ibid., p. 5.

(5)Ibid., p. 16.

(6) Simone MARCUSJEISLER, "Réponse à l'enquête sur les effets psychologiques de la guerre sur les enfants et les jeunes gens en France”, Revue de la sauvegarde, février 1947, pp. 3 à 24 .

(7) René BIARD, $o p$. cit., p. 30.

(8) Ibid., p. 30.

(9) Claude CHARMES, Le maximum, Paris, Stock, 1974, p. 17. particulier dans les troubles de la délinquance juvénile et infantile. " ${ }^{(3)}$ Il signale aussi que, entre 1938 et 1944, "le nombre d'enfants vagabonds confiés à des cuvres privées a été multiplié par 6 ». ${ }^{(4)}$

Cette dissolution familiale, poursuit G. Heuyer, est accentuée par les 8 millions de mobilisés pendant près d'un an, les 1.951 .000 prisonniers, dont les $4 / 5$ resteront en captivité jusqu'en 1945 . A ces chiffres, il ajoute " 780.000 requis du STO, 200.000 déportés, 40.000 fusillés». ${ }^{(5)}$ "Les adolescents, du fait de l'absence de leur père restés en compagnie de leur mère, se sont montrés souvent autoritaires, violents, brutaux ». Le Dr Simone MarcusJeisler, dans une enquête de 1946 sur "Les effets psychologiques de la guerre sur les enfants et les jeunes gens en France ", ${ }^{(6)}$ insiste sur le lien qui existe entre la mobilisation et l'augmentation importante de dépôts d'enfants à l'Assistance publique entre août et octobre 1939, dont le chiffre a été multiplié par 3,5.

René Biard est placé très jeune en nourrice chez un oncle et une tante auxquels il va fortement s'attacher, mais à l'âge de 10 ans, en 1935, pour des raisons liées surtout à la mésentente entre son père et son oncle, il se retrouve placé à l'Institut Théophile Roussel à Montesson, qu'il décrit comme "une formidable école de redressement moral »."

Il s'agit d'une institution bien connue dans la région parisienne, qui fut ouverte à la fin du siècle dernier par le département de la Seine pour accueillir les enfants difficiles de l'Assistance publique et les mineurs de la correction paternelle. On ne sait guère ce que fut le statut d'enfant placé de René à Montesson. Il y restera jusqu'en mars 1940, date où son père demande à le reprendre. Ce retour chez le père sera de courte durée ; il y rencontre une belle-mère qu'il ne connaissait pas. Après quelques jours, d'un commun accord avec le père, René retourne chez l'oncle et la tante de son enfance. "C'est, nous dit-il, après 5 ans de Montesson un moment de bonbeur qui commence : Ca y est, je suis beureux ! ". ${ }^{(8)}$ René trouve rapidement un emploi de souffleur de verre dans une fabrique de lampes à Colombes, mais cela ne va guère durer.

Claude Charmes nous explique que ses parents ont divorcé dès la période de sa naissance : "J'ai été trimbalé de nourrices en institutions ". ${ }^{(9)}$ Il a très peu de relations avec son père, qui vit à Paris où il occupe un 
emploi de metteur au point chez Rolls Royce. Il évoque à peine sa mère, qui vit avec un autre homme. Il a une sœur qui est placée.

Au moment de la déclaration de guerre, en septembre 1939, Claude Charmes est placé en nourrice à Milly-la-Forêt, près de Fontainebleau, dans la famille du chef de gare.

\section{III - L'exode, les premiers mois de l'occupation, la rencontre avec la délinquance}

C’est une évidence de dire que la période de la guerre a contribué à l'augmentation de la délinquance juvénile. Les chiffres apportés par Henri Gaillac en sont une illustration : 12.165 décisions de Justice à l'égard des mineurs délinquants en 1939, près de 13.000 en 1940, 35.000 en 1942, plus de 40.000 en 1945. Ce chiffre ne commencera à décroître qu'après $1946 .{ }^{(10)}$

On peut dire de l'exode de mai-juin 1940 qu'il est, plus que la mobilisation, le premier grand traumatisme social de cette guerre. Il va toucher, selon W.D. Halls, ${ }^{(1)}$ près d'un cinquième de la population française, ce qui représente plusieurs millions d'enfants, parmi lesquels 90.000 enfants perdus ne retrouveront leurs familles qu'après plusieurs semaines, voire plusieurs mois, et parfois seulement après la guerre. Simone Marcus-Jeisler parlera d'enfants vivant en bande, " ayant perdu contact avec leurs parents pendant l'exode, errant en groupe dans les campagnes, vivant de rapine et de charité avant de se fixer quelque temps autour de grandes villes comme Toulouse, Lyon, Marseille». «Ils furent, ajoute Simone MarcusJeisler, rapidement dispersés et remis à des patronages ". ${ }^{(12)}$

Après juillet 1940, les internats de toute sorte, dont ceux de l'Éducation surveillée, regorgent d'enfants, de jeunes de toute provenance, alors que beaucoup des personnels de ces institutions sont sous les drapeaux. Des incidents graves éclateront le 18 juin 1940 à la Maison d'éducation surveillée de Saint-Hilaire, ${ }^{(13)}$ mais aussi à l'Institut Théophile Roussel de Montesson, que nous évoquerons dans le cadre de cet article..$^{(14)}$

Contrairement à beaucoup de jeunes français de l'époque, René et Claude n'auront pas leur père prisonnier et ne feront pas l'exode ; ils le verront passer. Ils font partie de ceux qui, malgré la menace de l'avan-

(10) Henri GAILLAC, Les maisons de correction, Paris, Cujas, 1972, p. 361.

(11) Wilfred Douglas HALLS, Les jeunes et la politique de Vichy, Paris, Syros, 1988, p. 19.

(12) Simone MARCUS-JEISLER, $o p$. cit., p. 8.

(13) "Nus dans leurs cellules, roués sous les coups, ils attendirent la mort pendant deux jours", écrit, le 14/12/40, l'envoyé spécial de Paris soir, Jean Steve.

(14) M. BLONDELPASQUIER, La maison d'éducation de Montesson, Mémoire de la Faculté de médecine de ParisXII, 1993. 
(15) Habitation à bon marché de la loi Sellier, qui préfigure les HLM.

(16) René BIARD, op. cit., p. 54.

(17) Ibid., p. 55.

(18) Henri AMOUROUX, Histoire des français sous l'occupation, Paris, R. Laffont, 1993, Tome 1, p. 414.

(19) Simone MARCUS-JEISLER, $o p$. cit., p. 7. cée allemande, restent chez eux. C'est toutefois à cette occasion que tous deux rencontreront une situation qui peut préfigurer la délinquance. Juin 1940, René perd son emploi. Il l'exprime dans un langage imagé : "Avec les Allemands qui rallègent, les tauliers ont fermé la grille. " Il va voir le marchand de journaux qui est en face de son HBM. ${ }^{(15)} \mathrm{Ce}$ dernier lui propose d'aller vendre des journaux au stade de Colombes où sont enfermés "des gens qui appartiennent à la Sème colonne ». L'exploit est d'entrer dans le stade. Cela ne dure qu'un jour ; le lendemain il n'y a plus de journaux et les détenus de la 5ème colonne ont été envoyés ailleurs. C'est l'exode : "Les habitants des HBM s'enfuient. " Ce n'est pas le cas de l'oncle de René qui pense que ça ne servirait à rien de partir : "on manquerait d'essence, ils sont plus rapides que nous ". ${ }^{(6)}$

René assiste à quelques pillages de magasins auquel il apporte sa collaboration : "Les gens alentours, bien probes, bien bonnêtes allèrent faire un tour au Familistère que les gérants avaient abandonné. [...] C'est par brouettes pleines que les provisions se sont fait la paire. [...] J'y vais aussi, mais je n'ai pas pris de précaution, je ne peux que remplir mes poches. [...] La conscience est tranquille disent les gens, [...] toujours ça que les boches n'auront pas. " ${ }^{(17)}$ Henri Amouroux évoque, dans son ouvrage sur l'occupation, de véritables pillages de magasins à Paris pendant les jours de l'exode. ${ }^{(18)}$ Très vite le problème de ravitaillement va devenir la préoccupation essentielle des familles, débouchant sur le système D et le marché noir. Le Dr Simone Marcus-Jeisler écrit : "Déjà pendant l'exode, les réfugiés ont vécu d'expédients, de pillages auxquels les enfants ont été associés. Le marché noir qui va se développer progressivement va devenir une nécessité qui est une désobéissance au gouvernement de Vichy, mais qui peut prendre aussi des allures patriotiques en détournant les produits des réquisitions allemandes. " ${ }^{(19)}$

René assiste à l'arrivée des troupes allemandes dans Paris ; un soldat lui demande d'aller lui chercher un carton de cigarettes Balto dans un bureau de tabac. René gardera les 5 marks. L'Allemand n'aura jamais ses cigarettes.

Premier acte de délinquance ? Premier acte de résistance ?

A 70 kilomètres plus au sud, à Milly-la-Forêt, Claude Charmes regarde passer le flot des réfugiés qui vient de Paris. Une voiture s'arrête, une femme lui demande où elle pourrait acheter du sucre et de l'huile. Le 
petit Claude se propose d'y aller. Il gardera la pièce de dix francs et l'automobiliste ne le verra jamais revenir.

La guerre, facteur de délinquance?

Claude nous apprend que, quelques jours plus tard, il a volé de l'argent dans la recette du chef de gare pour acheter des sunset gums (l'ancêtre du chewing gum). La réaction est rapide et violente : il se retrouve, malgré ses 8 ans, une journée et une nuit à la gendarmerie ; on le ramène à Paris, chez sa mère, où un beau-père, ancien sous-officier des spahis, l'accueille à coups de ceinturon.

Le retour chez la mère s'avère difficile. Les conflits avec le beau-père sont quasi quotidiens. Claude fait des séjours punitifs à la cave, que le beau-père qualifie de "gnouf". Régulièrement menacé de placement en maison de correction, suite à divers chapardages, Claude s'oppose de plus en plus, devant une mère indifférente. Seule l'école, où il est bon élève, demeure un lieu privilégié. Les menaces arrivent à exécution début 1941. Claude, âgé de 9 ans, est placé à l'école Théophile Roussel de Montesson, où René Biard vient de passer 5 ans.

René profite pleinement des premiers mois de l'occupation à Paris. Après avoir vendu pendant quelques jours le Petit Parisien, il change d'organe de presse et devient vendeur du Parizer Zeitung à Pigalle. Cela rapporte beaucoup plus et René découvre une première forme de collaboration.

Son commerce devient vite florissant sur l'axe Pigalle-Place Blanche. Il y adjoint la vente de souvenirs de Paris pour « une clientèle prête à acheter n'importe quoi à n'importe quel prix ".

René se fait bien arrêter deux ou trois fois par la police - il est mineur -, mais cela n'a pas de conséquences. Seuls son oncle et sa tante s'inquiètent de ses absences de plus en plus fréquentes et de son activité peu définie il lui arrive de plus en plus souvent d'être rabatteur pour des boites spécialisées de Pigalle - ; le commissaire de Police leur répond «Votre neveu ne fait pas l'imbécile, laissez-le faire. »C'est l'apprentissage du système $\mathrm{D}$ que découvre René, comme bien d'autres de ses contemporains.

La frontière entre le licite et l'interdit est de plus en plus floue.

Les choses vont toutefois se gâter en mai 1941. René explique que «les 
(20) Georges

HEUYER, op. cit., p. 7.

(21) René BIARD, op. cit., p. 64.

(22) Ibid., p. 85-86.

(23) Ibid., p. 91.

(24) Officiellement on parle d'IPES (Institution publique d'éducation surveillée) depuis la circulaire du 25/02/40 ; le terme MES restera le plus souvent employé, jusqu'à la création de la direction de l'Éducation surveillée en septembre 1945. affaires sont en perte de vitesse ". Le système $\mathrm{D}$ est légitimé. Il devient nécessaire. G. Heuyer parle "du renversement des valeurs morales ; [...] le mensonge, le vol ont pu être des vertus pendant la guerre ". ${ }^{(20)}$ Le marché noir devient une source d'enrichissement facile dont certains jeunes sauront profiter. Marcel Aymé décrit dans Le chemin des écoliers, roman écrit en 1946, de jeunes lycéens qui vivent fort bien du marché noir, tout en poursuivant la préparation de leur baccalauréat. Le retour à des valeurs plus conformes à la norme morale sera un des problèmes de l'après-guerre.

La clientèle se raréfie, nous explique René Biard. "Les militaires se font de plus en plus rares, le front de l'Est va en utiliser beaucoup. " ${ }^{(21)}$ Il rencontre un ancien de Montesson. Tous deux vont se faire prendre pour un vol à l'arraché en plein boulevard de Clichy, le 31 mai 1941. Après une nuit au commissariat, René découvre le quartier des mineurs de Fresnes, où il est placé en détention provisoire. Il est à la 4ème division, celle des mineurs. C'est, dit-il, " une prison de malades, de malades qui meurent de faim. Chez les mineurs, c'est un désastre ". Il a quelques idées sur la notion de l'honnêteté : "Elle est une vertu d'homme ayant dîné. [...] Le marché noir est roi, tout se vend, s'achète, se troque [...] et les mois de cabane dégringolent : le charbon vaut 8 mois de tôle, le sucre 13 mois, les pneus de vélo 15 mois..." ${ }^{(22)}$

Fresnes, c'est aussi pour René le souvenir des otages de la 1ère division : "Ils chantaient la Marseillaise et l'Internationale à heures fixes. " ${ }^{(23)}$

Le 27 septembre 1941, René comparaît devant le tribunal pour enfants et adolescents de la Seine : son père est présent, bien qu'il travaille en Allemagne où il a un emploi. Il propose d'y emmener son fils. Ce ne sera pas le choix du tribunal. René est acquitté en tant que mineur non discernant et placé jusqu'à sa majorité dans une "Maison d'éducation surveillée" (MES). ${ }^{(24)}$

Cette décision demande une explication. On peut être étonné de cet acquittement en tant que mineur non discernant pour un garçon de 16 ans. En 1941, la loi de 1912 sur l'enfance délinquante est toujours en vigueur, qui distingue les mineurs discernants et les mineurs non discernants pour lesquels le tribunal peut choisir une mesure d'éducation. C'est ce dernier choix qui sera fait pour René, le tribunal atténuant ainsi sa responsabilité pénale. Un an plus tard, l'acte dit "loi du 27 juillet 
1942" sur l'enfance délinquante mettra fin à cette notion de discernement pour la remplacer par celle d'éducabilité du mineur délinquant. La voie éducative sera la voie privilégiée, mais elle en exonérera le plus souvent les mineurs de 16 ans, pour lesquels l'excuse de minorité sera rarement envisagée.

Le tribunal pour enfants et adolescents de la Seine envoie René Biard à la Maison d'éducation surveillée de Belle-Ile-en-Mer en vue de sa rééducation et de sa formation professionnelle.

\section{IV - Le temps des internats}

\section{Entre correction et rééducation, 1941-1944}

\section{- René à la MES de Belle-Ile-en-Mer, puis à celle de Saint-Maurice}

Belle-Ile est une ancienne colonie pénitentiaire et maritime construite en 1880, à vingt kilomètres de la côte, pour les mineurs délinquants. Depuis 1927, elle porte le nom de Maison d'éducation surveillée. On a changé le nom, mais le projet reste très répressif et pénitentiaire. C'est suite à une révolte des pupilles de Belle-Ile, en août 1934, que débutent les campagnes de presse contre les bagnes d'enfants et les premières velléités de réforme de ces institutions.

En 1941, la MES de Belle-Ile n'a guère profité de ces réformes ; l'univers que nous dépeint René tient plus du pénitencier que de la maison d'éducation. Il est toutefois surpris de l'accueil des surveillants qui souhaitent qu'on les appelle "père", par son numéro de matricule. René est affecté au dortoir marine, " 60 cages grillagées de $1 \mathrm{~m} 90$ sur $1 \mathrm{~m} 10$, sur la porte desquelles figure le numéro de matricule du pensionnaire ». Il a le souvenir de l'homosexualité ambiante, la relation des "mignons" et des "caïds" : "Il faut se défendre pour ne pas être macqué. " Le climat maritime ne lui convenant guère, c'est pour des raisons médicales que René quittera Belle-Ile en février 1942. Il aura l'impression de n'y avoir rien appris et conclura dans son langage de petit truand : "Le tribunal s'est mis la balance dans l'ceil en m'envoyant à Belle-Ile. . ${ }^{(26)}$

Il est à remarquer que ce départ de Belle-Ile correspond aussi à un vidage progressif de l'institution. L'île était devenue un point stratégique

(25) Ibid., p. 122.

(26) Ibid., p. 150. 
(27) Cité par Philippe REY-HERME, Quelques aspects du projet pédagogique dans la rééducation de la jeunesse délinquante, Paris, VRIN, 1945, p 186.

(28) On les appelle moniteurs depuis 1927. On parle de moniteurs éducateurs à partir de 1938.

(29) René BIARD, op. cit., p. 158. "Ausweiss" : laissez-passer. du mur de l'Atlantique et la majorité des pensionnaires seront envoyés, début 1942, à la MES de Saint-Maurice, qui sera la destination de René.

L'accueil à Saint-Maurice, René en a gardé un souvenir ambigu : "On est accueilli dans la cuisine, tout respire la propreté, [...] je me régale; il y a bien longtemps que je n'ai rien mangé d'aussi bon, [...] mais ici on ne dit pas père, on dit monsieur" et, pour ne pas l'avoir dit, René reçoit sa première “dérouillée". C'est la mise en condition.

Saint-Maurice, ouvert en 1872, a la même histoire que Belle-Ile ; c'est une ancienne colonie pénitentiaire. Saint-Maurice a néanmoins cet avantage d'avoir été, en 1937-1938, un des fleurons de la réforme des établissements pour mineurs. Malgré les archaïsmes pénitentiaires qui subsistent, l'administration a fait appel, dès avant la guerre, à la Direction de l'enseignement technique pour poser les bases de la réforme. Saint-Maurice, qui reçoit toujours des mineurs placés par les tribunaux, se modélise, depuis 1938, sur l'image de l'école professionnelle.

Les réformateurs de 1938, qui dirigent toujours l'établissement en 1942, ont écrit en 1939 : "On ne peut dire que les principes de l'éducation des pervertis, des délinquants soient différents de ceux des garçons normaux; ils sont souvent les mêmes et ne varient que dans leur application. " ${ }^{(27)}$ Il s'agit de réintroduire le jeune délinquant dans une normalité éducative, ce qui se conçoit bien dans l'esprit de la loi du 27 juillet 1942 et des projets qui l'ont précédée.

René Biard a plutôt un souvenir positif de Saint-Maurice : "Rien n'est ici comparable à Belle-Ile, la propreté y règne, la ferme bienveillance de nos surveillants ${ }^{(28)}$ me fait admettre que je suis plus un pensionnaire qu'un détenu. " Suite à sa demande, René Biard est affecté à la formation de menuisier. Au bout de neuf mois, si son comportement et ses résultats scolaires ont été bons, l'élève (on ne dit plus pupille) aura droit à une permission de dix jours. Début 1943, René a obtenu sa permission ; il se retrouve à Colombes chez son oncle et sa tante. Au cours d'une balade à Pigalle, il rencontre un souteneur qu'il a connu en 1941 et qui lui propose de travailler avec la Gestapo pour « participer à des filatures, à la recherche de ceux qui troublent l'ordre, qui se planquent. [...] Tu auras du pognon, un ausweiss $"{ }^{(29)}$ René ne donne pas suite et rentre à Saint-Maurice. La guerre 
se poursuit ; "On chante Maréchal nous voilà en traversant en rang les rues de la Motte-Beuvron. ${ }^{(30)}$ Le sport a une place de choix ; après l'apprentissage, c'est la seconde priorité de "l'Ecole de Saint-Maurice" ; René ne s'y adonne guère.

Philippe Rey-Herme, jeune jésuite qui soutient, en avril 1944, à l'Université de Paris, une thèse de doctorat ès lettre sur la rééducation de la jeunesse délinquante, évoque longuement, dans l'ouvrage qu'il en a tiré, la réforme de Saint-Maurice : "Il ne s'agit pas de mettre les jeunes délinquants dans l'impossibilité de nuire, [...] mais de faire d'eux des membres normaux de notre société, [...] adapter à la vie normale d'une communauté bumaine des enfants qui, par suite d'une mauvaise éducation, d'une bérédité chargée, de troubles plus ou moins innés ou simplement d'une volonté trop faible, se sont laissés aller à des actes anti sociaux. " ${ }^{(31)}$

L'image de l'enfant délinquant, celui que l'on appelle l'enfant coupable en 1937, s'apparente aussi à l'enfant victime de sa famille, de sa mauvaise éducation. Ce mal éduqué, il faut l'enlever à sa famille, envisager sa rééducation en internat. Seuls les plus pervertis, dont la notion est assez mal définie, peuvent relever de la sanction.

Le directeur de Saint-Maurice affirme que "plus que la formation morale, la formation professionnelle constituera le soubassement de l'œuvre de rééducation $»{ }^{(32)} \mathrm{Ph}$. Rey-Herme réagit à «cette affirmation, qui, écrit-il, n'est pas sans danger». Il évoque ce jeune qu'il a rencontré, qui sort d'une maison d'éducation surveillée avec un CAP de serrurier en poche ; il est félicité par le sous-préfet qui le donne en exemple à ses camarades. A peine descendu de l'estrade, le nouveau diplômé se tourne vers ses camarades : "A nous maintenant les coffres-forts Fichet. " ${ }^{(33)}$

On a beaucoup parlé de "remoralisation" de la jeunesse sous Vichy. Qu'en était-il de l'action morale pendant la guerre à Saint-Maurice ? "La morale est action, écrivait V. Hourcq, le directeur, l'homme se forme moralement par ses actes [...] jusqu'à ce que bien agir devienne non seulement une babitude, mais une joie. " ${ }^{(34)}$

Différemment, Ph. Rey-Herme lie l'éducation morale à l'idéal et se réfere en éducation aux techniques du scoutisme. Les deux propos se rejoignent et sont constitutifs des projets de rééducation de mineurs

(30) Ibid., p. 162. L'IPES était implantée sur la commune de la Motte-Beuvron dans le Loir-et-Cher.

(31) Philippe REYHERME, op. cit., p. 146-147.

(32) Ibid., p. 186. (33) Ibid., p. 187. (34) Ibid., p. 186. 
(35) 21 ans.

(36)René BIARD, op. cit., p. 165.

(37) Archives de SaintMaurice, CNFEPJJ, Vaucresson.

(38) Témoignages enregistrés de René Courtois, directeur de Saint-Maurice en 1944, et de R. Chaumorcel, élève à Saint-Maurice en 1944, CNFEPJJ, Vaucresson, 1986 et 1995.

(39) Claude CHARMES, op. cit., p. 126.

(40) Ibid., p. 31 délinquants qui se développent pendant la guerre et au lendemain de la guerre : une pédagogie centrée sur le groupe de jeunes, qui ira dans un premier temps chercher ses références dans les mouvements de jeunesse et plus particulièrement dans le scoutisme.

En mai 1944, René réussit son CAP de menuisier. Quelques jours plus tard, c'est le débarquement, une nouvelle qui va fortement perturber les élèves de Saint-Maurice : va-t-on nous libérer ? faudra-t-il attendre la vingt-et-une ? ${ }^{(35)}$ va-t-on remettre en cause toutes les décisions de Justice ? ${ }^{(36)}$ Les questions sont sans réponse.

René a son CAP, ce qui lui donne une permission de dix jours. En juillet 1944, il est à Colombes, à Paris ; il oubliera de revenir ; il ne sera pas le seul. Les archives de Saint-Maurice que nous avons consultées ${ }^{(37)}$ et les quelques témoignages oraux que nous avons eus ${ }^{(38)}$ laissent entendre que les fugues de Saint-Maurice et les non-retours connurent, entre juillet et août 1944, une très sensible augmentation.

\section{- Claude à Montesson}

9 ans, c'est bien jeune, pour se retrouver à l'Institut Théophile Roussel à Montesson. Le directeur qui l'accueille laisse entendre à Claude qu'il en aura jusqu'à 21 ans : "trois fois le temps de mes souvenirs les plus éloignés ", ${ }^{(39)}$ pense intérieurement le jeune pensionnaire.

Montesson, institution laïque par excellence, ouverte en 1895, a donné à ses pavillons des noms qui se réferent à l'esprit des lumières et à la République : "La Fontaine, Jean Macé, Diderot, d'Alembert, Condorcet, Victor Hugo, Michelet ». Pourtant, comme l'écrit Claude Charmes, "l'esprit ne semblait guère souffler sur cette institution ". 400 élèves répartis suivant leurs aptitudes scolaires. Claude, qui à 9 ans était déjà dans la classe du certificat, se retrouve à Montesson avec des pensionnaires de 14 ans.

Un autre pavillon, appelé Ambroise Rendu, du nom d'un édile parisien, mais aussi le "petit château", sert d'isolement punitif. Claude y a fait des séjours : "Ony dort, on s'y masturbe ", ${ }^{(40)}$ mais, précise-t-il, "j’y répétais inlassablement toutes les récitations que je savais".

Il est le premier de sa classe. Le jour de sa première communion, il reçoit les félicitations du cardinal Suhard, archevêque de Paris. Mais le 
climat de Montesson ne lui convient guère et ses fugues succèdent aux fugues. L'une d'elles laissera un souvenir durable dans l'institution : il saute du Pont du Pecq dans la Seine pour échapper aux poursuivants ; il sera repêché par des mariniers et son retour à Montesson en fera une vedette.

Les fugues de Claude se multiplient. Elles se font à plusieurs. L'une d'elles, en 1943, durera trois semaines du côté de Bagnolet au nord de Paris : "C'était la belle vie, la liberté, pas de problèmes; [...] nous avions fabriqué une cabute avec des planches et de la tôle goudronnée. Après avoir cambriolé quelques boutiques, on partageait nos larcins en frères, une quatrième part servait au troc le lendemain pour se procurer des cigarettes. " ${ }^{(1)}$

Ce sont les gitans qui alertèrent la police et les Allemands ; le lendemain ils étaient de retour à Montesson. "Début 1944, à Montesson, on ne nous donnait presque plus rien à manger, [...] tous les mômes maigrissaient à vue d'ail, je volais le mélassé que l'on distribuait aux chevaux de la ferme, cela calait l'estomac. " ${ }^{(42)}$

L'ultime fugue, celle qui réussit, eut lieu au printemps 1944, peu avant le débarquement.

\section{- En cavale, la liberté. Le temps de la Libération}

La période qui précéde le débarquement et la Libération n'est pas sans évoquer un climat de désorganisation progressive du pays, voire même de "guerre civile", comme l'évoque Gilles Ragache ${ }^{(43)}$ Elle amènera à la constitution de bandes d'enfants laissés à eux-mêmes, vivant dans une sorte de no man's land de la loi. ${ }^{(4)}$ Parfois, délinquance et actions héroïques se joignent. Le directeur de Saint-Maurice, en 1944, évoque deux fugueurs de son institution qui seront tués sur les barricades à Paris pendant les journées de la Libération. Des héritiers de Gavroche...

René Biard et Claude Charmes vont vivre "en cavale" ces moments forts de l'histoire du pays. René ne retourne pas à Saint-Maurice. Il s'installe à Pigalle. Il est à nouveau contacté par deux français de la Gestapo qui s'apprêtent à quitter Paris en lui proposant de les accompagner. René plus lucide que patriote leur répond "qu'il a plus de confiance en ceux qui viennent que dans les Fritz qui décarrent". Il devient chasseur dans une boîte de nuit, couche dans les hôtels de passe sous la protection d'une

\author{
(43) Gilles RAGA- \\ CHE, Les enfants de la \\ guerre, Paris, Perrin, 1997, \\ p. 21.
}

(44) Lire AISHA, Décharge publique, Maspéro, 1980, p. 29 et suivantes. 
(45) René

BIARD, op. cit., p. 185.

(46) GI : abréviation américaine de Government Issue ; nom donné aux soldats de l'armée américaine à partir de 1942.

(47) Ibid., p. 192.

(48) Ibid., p. 244. mère maquerelle qui l'a pris sous sa coupe. Le 19 août, les murs de Paris sont couverts d'affiches. La libération approche. On lui dit que le Palais de Justice est en flammes. René se rend sur place et, déçu, voit qu'il n'en est rien : "Les miliciens cavalent après les FFI, les FFI après d'autres mecs", écrit-il. Il se fait arrêter, montre sa permission de Saint-Maurice. On lui propose de s'engager dans les FFI. Il reste extérieur au combat de la Libération; il ne sera pas un partisan. Il renvoie dos à dos les anciens collabos et les nouveaux libérateurs, "ceux qui l'ont foutu au trou pendant dix ans ". ${ }^{(4)}$ Blasé, René commente les événements loin des idéaux du moment : "Ceux qui hier goûtaient la joie d'être les plus forts s'en vont aujourd'bui mitraillette dans le dos, [...] cela me fait doucement marrer. " "Avec l'arrivée des Américains, poursuit René, les $G I^{(46)}$ prennent le baut du pavé avec leurs dollars. [...] Il n'y a pas de travail mais du fric partout. " ${ }^{(47)}$

René se lance alors dans le trafic de faux billets : "J'achète 50 billets de cent francs, $j$ 'aurai un bénéfice de quatre mille francs. " L'affaire est risquée. Il se fait très vite arrêter, pour se retrouver à Fresnes, puis à Saint-Maurice où il est mis au mitard. René aura alors le temps, ainsi qu'il l'écrit, de faire le compte de son temps d'enfermement : depuis le 17 mars 1935, date de son arrivée à Montesson, " 129 mois, 3870 jours, 92880 beures, parce qu'à l'âge de 10 ans mon père et ma mère ne s'entendaient pas " ${ }^{(48)}$

Fin mars 1944, Claude tente, avec deux camarades, sa dernière cavale de Montesson, celle qui réussira. Elle les conduit à Saint-Germain-enLaye, puis à Mantes où, pendant quelques jours, ils vivent sur une petite île de la Seine une vie de Robinson. Début avril 1944, ils sont à Caen et se placent chez des agriculteurs, auxquels ils racontent qu'ils sont frères et que leurs parents ont été tués pendant les bombardements du quartier de la Chapelle à Paris. Ils ne réclament que le gîte et le couvert et on ne leur pose pas trop de questions ; en échange ils doivent fournir un travail qui, au fur et à mesure des jours, se révèle de plus en plus dur. Ils partent, pour se faire arrêter deux heures plus tard à l'octroi de Caen. Ils sont confiés par la police à un orphelinat, où le directeur de Montesson devrait venir les chercher. L'épisode de Caen ne dure que quelques jours. Après un incident, Claude et un de ses deux compagnons de fugue partent vers Ouistreham sur la côte, où ils sont héber- 
gés par des soldats allemands cantonnés près des blockhaus du Mur de l'Atlantique. Le 6 juin, ils sont aux premières loges : "La terre tremble, les canons tirent à la volée, des parachutistes, c'est le débarquement. " Les deux garçons se réfugient dans une cave. C'est un parachutiste qui les retrouvera le soir dans les décombres de la maison. C'est la première cigarette américaine, le chewing gum, bien meilleur que le sunset gum de Millyla-Forêt, précise Claude.

Les deux garçons se séparent, Claude devient la mascotte d'un groupe de soldats anglais avec lesquels il met deux semaines pour arriver à Caen. Il constate alors que l'orphelinat avait "été rasé par un tapis de bombes". "J'ai pensé, ajoute-t-il, que la chance est revenue. " ${ }^{(49)}$

Les semaines qui suivent, Claude les intitule "la grande vadrouille d'un Gavroche ». Il porte un blouson américain, un pantalon et des bottes de saut canadiennes, on l'appelle "Frenchie" ou "Boy". " ${ }^{(50)}$ "Ce sont mes premières vacances", écrit Claude. Il découvre "l'opulence et la liberté" au milieu "du bordel indescriptible de la libération". ${ }^{(51)}$ Il passe des anglais aux américains, aux canadiens. C'est avec eux qu'il arrivera à Paris au moment de la libération de la ville, pour échouer dans un centre de réfugiés à Saint-Mandé, où il raconte qu'il est belge et que ses parents ont été déportés. Il voit les dernières barricades de l'insurrection parisienne, assiste "en badaud insensible et un peu morbide à l'exécution d'un milicien qui suait de peur ". ${ }^{(52)}$

L'épisode de Saint-Mandé ne va pas durer longtemps. Claude ne souhaite revoir ni sa mère, ni son beau-père. Il part vers Luna Park ${ }^{(53)}$ à la Porte Maillot, où des forains lui proposent de faire le rabatteur auprès des soldats américains. René Biard, 4 ans plus tôt, l'avait précédé dans des activités de ce type. Puis il se fait arrêter sur les Champs-Elysées : "Les agents n'ayant plus de miliciens à rattraper, ils redeviennent soupçonneux. " ${ }^{(54)}$ Le retour à l'ordre social ?

Il a 12 ans. Le directeur de Montesson n'en veut plus. On l'envoie à l'hôpital Sainte-Anne, au pavillon Esquirol, "où j'aiderai les infirmières ". En décembre 1944, Claude est confié à l'Assistance publique : "J'étais, écrit-il, avec les sans famille et les enfants trouvés. Ma famille m'avait abandonné et j'étais désormais un gamin perdu. " ${ }^{(55)}$
(49) Claude

CHARMES, op. cit., p. $41-42$.

(50) Ibid., p. 41-42.

(51) Ibid., p. 47.

(52) Ibid., p. 48.

(53) C'était un grand parc d'attractions.

(54) Ibid., p. 50.

(55) Ibid., p. 51. 
(56) Ibid., p. 56.

(57) Ibid., p. 62.

\section{V - Épilogue}

La guerre se termine. René Biard est presque majeur. Il attendra toutefois la "vingt-et-une ", en mai 1946, pour quitter Saint-Maurice et retrouver la liberté. Liberté bien éphémère, puisque, en 1951, il sera condamné à dix ans de travaux forcés.

Pour Claude Charmes la fin de la guerre correspond à son placement à l'Ecole de réforme de Chanteloup, une institution pénitentiaire qui reçoit, depuis le début du siècle, les mineurs de moins de 14 ans.

C'est dans cet établissement, qu'il qualifie "d'école de la paresse et d'abrutissement ", qu'il renoue avec "ses rêves et sa révolte ": " 60 gosses de 10 à 14 ans réputés difficiles que le directeur considérait comme pas bons à grand chose. ${ }^{(56)}$

La création de la direction de l'Éducation surveillée, en septembre 1945, va amener à Chanteloup un changement que Claude qualifie de "radical". Un couple d'enseignants, venant de l'Éducation nationale, remplace la direction pénitentiaire de l'établissement. Monsieur et Madame Vincendon, disciples d'Adolphe Ferrière, un des pionniers de l'éducation nouvelle, sont chargés par la nouvelle Éducation surveillée, qui vient de conquérir son autonomie sur l'Administration pénitentiaire, de réformer Chanteloup.

Claude écrit : "Nous sommes traités comme des enfants normaux. " ${ }^{(57)} \mathrm{Il}$ retrouve les activités scolaires où il réussit si bien, il découvre la musique, des rudiments de scoutisme avec les jeunes élèves officiers de l'école de cavalerie de Saumur qui viennent faire du "social" à Chanteloup. "Ce furent, écrit Claude, 18 mois de détente, de jours heureux, sans histoire et pas une seule tentative d'évasion; [...] d'ailleurs nous aurions pu partir par la porte, elle était constamment ouverte."

L. Vincendon, dans un ouvrage qu'elle a consacré à Chanteloup, évoque Claude Charmes :

"Il nous arriva un beau matin précédé d'une fâcheuse réputation. [...] Il a l'air droit, énergique. [...] Il a déjà l'air d'un homme absolu, capable du meilleur comme du pire, nous choisissons le meilleur. [...] Il a une volonté extraordinaire, capable d'extrêmes opposés, mais pas de choses moyennes. Sa mère n'a pardonné aucune de ses erreurs enfantines. Il n'a peur d'aucune souffrance, un 
entrain endiablé, $[. .$.$] un désir vorace d'apprendre, [. .$.$] mais il nous donnait$ des inquiétudes dans ses rapports avec des adultes peu compréhensifs, indifférents. [...] Nous ne l'avons eu qu'une seule année. " ${ }^{(58)}$

Ce projet de Chanteloup, un des plus audacieux de l'Éducation surveillée de l'après-guerre, sera éphémère et ne durera que jusqu'en 1951. Il ouvrait peut-être trop de nouveaux horizons pour les enfants les plus jeunes de l'Éducation surveillée et ne les préparait pas à passer, à 14 ans, à l'IPES voisine de Saint-Hilaire, dont le régime était beaucoup plus dur.

Un nouveau texte a été promulgué, le 2 février 1945, sur l'enfance délinquante. Il s'inscrit dans la continuité de la loi de 1942, mais il apporte un élément complémentaire essentiel : la création du juge des enfants.

Le juge Chazal, un des tout premiers juges des enfants en 1945, écrivait, en évoquant les jeunes qui passaient devant lui : "Il faut atteindre chaque sensibilité, chaque intelligence dans leurs tendances et leurs aptitudes particulières. [...] La liberté dans l'action est la condition même des méthodes d'éducation nouvelle, elle suppose la confiance. ${ }^{(59)}$ La confiance, c'est peut-être la première fois que Claude la rencontre. Malheureusement, on ne peut rester à Chanteloup que jusqu'à 14 ans. C'est ce même juge Chazal qui le reçoit dans son cabinet, à Paris, en 1946, pour lui trouver une nouvelle solution.

Les parents refusent, malgré la demande du juge Chazal, de reprendre Claude chez eux. C’est une immense déception pour le jeune garçon : "Je n'entendais plus le juge. Finis les châteaux en Espagne. Quel idiot j’avais été de croire à la douceur d'un foyer! Moi,j'avais changé, mais mes parents étaient toujours les mêmes. " ${ }^{(0)}$ Après deux placements successifs au Patronage Rollet à Paris, puis dans un foyer à Bois-le-Roi, c'est, écrit Claude, «le retour du cycle infernal : délit, évasion, gendarme, orphelinat ". ${ }^{(6)}$

Ce ne sera pas l'orphelinat. Claude a aggravé sa situation judiciaire après un séjour à Fresnes, puis dans un hôpital psychiatrique. Il se retrouve à l'institution corrective d'Aniane, une ancienne maison de correction que la direction de l'Éducation surveillée n'a pas encore réformée et où l'on reçoit les mineurs les plus difficiles, dont les criminels.

Claude sortira d'Aniane en 1952, à 20 ans, avec un CAP de mécanique générale qui lui permet de réussir un concours dans l'armée de
(58) L. VINCEN-

DON, Chanteloup, Blainville-sur-Mer, L'amitié par le livre, 1979, p. 264.

(59) Jean CHAZAL, Les enfants devant leur juge, Paris, Ed. Familiales françaises, 1946, p. 56.

(60) Claude

CHARMES, op. cit., p 66.

(61) Ibid., p. 68. 
(62) René BIARD, op. cit., p. 248.

(63) Claude CHARMES, op. cit., p 236.
l'Air. Un vol de moto fera capoter ce projet. L'armée de l'air ne veut plus de lui et Claude s'engage dans un régiment de tirailleurs sénégalais, d'où il se fera réformer au bout d'un an.

René Biard et Claude Charmes connurent, après leur adolescence, de longues années de prison. Les vertus de la rééducation dont ils avaient fait l'objet restaient encore à prouver.

Nous ne pouvons avoir accompagné si longtemps ces deux jeunes dans leurs pérégrinations de la guerre en ignorant le message d'adulte qui donne sens à leur travail biographique.

René Biard conclut son livre par une sorte d'appel à la société avec l'espoir que "tous les enfants perdus, les petits copains à la recherche de leur vie d'bomme, tous les mineurs délinquants puissent ne pas endurer ce que nous avons dî endurer $"{ }^{(62)}$

Claude Charmes, s'il écrit ne pas vouloir délivrer de message, lance " un dernier cri d'angoisse à tous ceux qui ne savent pas se faire entendre : personne n'a le droit de dire à un homme, même s'il a commis un crime abominable, vous êtes irrécupérables. J'étais potentiellement irrécupérable, ce que j’ai réussi d'autres peuvent y parvenir, il faut les aider en leur donnant des motivations "; "La stimulation, conclut-t-il, c'est le moteur de l'action. ${ }^{(33)}$ 